\title{
Editorial
}

\section{Nueva estructura del Comité Editorial}

Como ya comenté en la editorial de diciembre de 2017, este año 2018 la revista Óptica Pura y Aplicada también cumple su 50 aniversario. Ya entonces anuncié algunos cambios en la gestión de la revista y en el Comité Editorial que, a mi modo de entender, profesionaliza la revista y la sitúa la revista al nivel de gestión, y espero que, de prestigio, de otras revistas científicas.

La travesía de la revista Óptica Pura y Aplicada desde una revista clásica a una revista que cumple con los estándares de las publicaciones más prestigiosas empezó bajo la gestión del anterior Editor Ignacio Moreno. Bajo su supervisión, la revista se digitalizó, se estabilizó la periodicidad de la publicación y se consiguió su inclusión en algunas de las bases de datos más utilizadas del mundo científico. Este trabajo ha tenido sus frutos años después ya que dotó a la revista de un prestigio que abrió la puerta a un flujo continuado de trabajos permitiendo pasar de la mera subsistencia a mirar el futuro con optimismo y a planificar con meses de antelación el flujo de trabajo.

Este éxito ha tenido tres consecuencias importantes. La primera es que, como ya anuncié en la última editorial del pasado año, la gestión de la revista ha desbordado las capacidades del editor y se ha procedido a una remodelación completa del Comité Editorial. Con la colaboración de los diferentes comités y de la Junta de Gobierno, se han nombrado una serie de Editores Asociados especializados en diversos temas que aparte de aligerar la gestión de la revista permiten un tratamiento más especializado de los temas (http://www.sedoptica.es/Menu Volumenes/editorial.php) Durante los seis meses que llevamos este año, hemos ido conociéndonos y acostumbrándonos a los nuevos modos de gestionar la revista. Hemos intentado integrar en este equipo a gente joven y experta, españoles e iberoamericanos y, con todas las dificultades que conlleva la creación de un nuevo equipo, he de decir que estoy más que satisfecho con el trabajo realizado en el poco tiempo que llevamos funcionando. Por supuesto que todavía existen deficiencias y despistes inherentes al uso de una nueva herramienta, pero esperamos ir superándolos con la ayuda y comprensión de todos.

La segunda consecuencia es menos visible, aunque no por ello menos importante. La gestión descentralizada de la revista ha impuesto la creación de una base de datos común de artículos y revisores, así como un nuevo sistema de envío de trabajos a través de una plataforma de gestión integral. Mediante este sistema tratamos de ser transparentes en la gestión de artículos, de modo que el autor podrá comunicarse con el editor asociado a su trabajo y comprobar el estado de su trabajo. De nuevo, cuesta acostumbrarse a las nuevas herramientas y todavía se producen ciertas disfunciones, pero estoy seguro de que, en breve, todo funcionará de manera óptima (http://sedoptica.es/OPAGestion/login.php).

La tercera consecuencia es, posiblemente la menos grata. El sistema de gestión de la revista requiere de unos gastos que la Sociedad Española de Óptica ha asumido con gran esfuerzo y a costa de otras 
partidas. Por ello, en la Asamblea del año 2017 se planteó la posibilidad de cobrar cuotas de publicación a aquellos autores no asociados a la Sociedad Española de Óptica No se trata de una medida recaudadora, ya que la tasa solicitada máxima solicitada de $50 €$ simplemente trata de cubrir ciertos costes ordinarios de la revista. (http://www.sedoptica.es/Menu_Volumenes/autores.php). Todos los trabajos enviados desde junio del año pasado han sido informados y esperamos que esto no sea impedimento para el envío de publicaciones.

Con estos cambios esperamos, por un lado mejorar la gestión de la revista y agilizar los procesos de recepción, revisión t publicación. Por otro, ir formando un equipo de trabajo que nos permita estar preparados para abordar los retos que nos esperan en el futuro.

Gracias a todos, editores, autores lectores y sobre todo, a la Sociedad Española de Óptica por confiar en nuestro trabajo.

\section{David Mas}

Editor Óptica Pura y Aplicada

Julián Espinosa

Secretario de Edición

DOI: http://dx.doi.org/10.7149/0PA.51.2.i 